\title{
UTILIZACIÓN DE COMPUESTOS QUÍMICOS PARA MANTENER LA CALIDAD EN PRODUCTOS MINIMAMENTE PROCESADOS DE PAPA
}

\author{
L. M. García-Procaccini' ${ }^{1}$ M.C. Monti² ${ }^{2}$ M. Huarte ${ }^{3}$
}

\section{Manuscrito recibido: 20/03/2014}

Aceptado: $28 / 10 / 2014$

Disponible en línea: Diciembre 2014

\section{Resumen}

La demanda por el agregado de valor a los productos de origen agropecuario ha generado interés sobre la preservación de productos mínimamente procesados (PMP). La vida útil de los PMP es disminuida por la reacción de pardeamiento enzimático, la cual causa una pérdida en la calidad del alimento. En el presente trabajo se estudió el efecto de la adición de compuestos químicos sobre el contenido de ácido ascórbico, fenoles totales, la actividad de la enzima PPO y el color de los PMP de papa (Solanum tuberosum L.). Los tratamientos fueron combinaciones de diferentes concentraciones de metabisulfito de sodio (MS), ácido ascórbico (AA) y ácido cítrico (AC). El ensayo se realizó en dos variedades de papa, Spunta y Newen INTA. Los tratamientos con $1,5 \%$ de AA y $1 \%$ AC mantuvieron la luminosidad y aumentaron la concentración de ácido ascórbico y el contenido de fenoles totales en el producto durante los primeros 10 días de almacenamiento. Es posible disminuir la concentración de MS si se lo combina con AA para mantener los parámetros de calidad en papas cortadas refrigeradas.

Palabras claves adicionales: Solanum tuberosum, antioxidantes, ácido ascórbico, ácido cítrico, fenoles, polifenoloxidasa.

1 Lic. en Ciencia y Tecnología de alimentos. Becaria CIC. Facultad de Ciencias Agrarias UNMdP. Ruta 226 km. 73,5 Balcarce, Argentina. garciaprocacciniluz@ gmail.com 2 Profesor Adjunto, Facultad de Ciencias Agrarias UNMdP. Ruta 226 km. 73,5 Balcarce, Argentina.

3 Investigador, Instituto Nacional de Tecnología Agropecuaria (INTA), Balcarce, Argentina. 


\title{
USE OF CHEMICALS TO MAINTAIN QUALITY POTATO MINIMALLY PROCESSED PRODUCTS
}

\begin{abstract}
Summary
Demand for value addition to the products of agricultural origin has generated interest in the preservation of minimally processed products (PMP). The shelf life of the PMP is decreased by the enzymatic browning reaction, which causes a loss in the quality of food. In this study the effect of chemical addition on the content of ascorbic acid, total phenolics, the PPO enzyme activity and colour of PMP potato (Solanum tuberosum L.) was studied. Treatments were combinations of different concentrations of sodium metabisulfite (MS), ascorbic acid (AA) and citric acid (CA). The assay was performed in two potato varieties, Newen INTA and Spunta. Treatments with $1.5 \%$ AA and $1 \%$ AC kept the brightness and increased the concentration of ascorbic acid and total phenolic content in the product during the first 10 days of storage. May decrease the concentration of MS when combined with AA for maintain the quality parameters in refrigerated potatoes cuts.
\end{abstract}

Additional keywords: Solanum tuberosum, antioxidants, ascorbic acid, citric acid, phenols, polyphenol oxidase. 


\section{Introducción}

En los últimos años se ha incrementado la demanda por el agregado de valor a los productos de origen agropecuario y esto ha generado interés sobre los procesos de preservación de los productos mínimamente procesados (PMP). Los consumidores demandan alimentos frescos, saludables, con calidad nutricional, de fácil preparación, con inocuidad química y microbiológica y que a su vez tengan una larga vida útil en el anaquel para facilitar la llegada al consumidor.

Si bien todos los vegetales son importantes en la dieta humana, algunas especies se destacan especialmente por su riqueza en nutrientes tales como vitaminas, minerales y fibras y resultan especialmente atractivos presentados como mínimamente procesados, tal es el caso de la papa. Uno de los mayores problemas ocasionados por el procesado mínimo de papa es el pardeamiento enzimático (Moretti et al., 2002; Petri et al., 2008; Yurong et al., 2010), el cual se genera luego de la ruptura de la integridad celular debida al corte, al entrar en contacto los polifenoles con el oxígeno. Esta es una reacción catalizada por la enzima polifenoloxidasa (PPO) que lleva a la formación de polímeros coloreados, de alto peso molecular, llamados melaninas, lo cuales ocasionan una pérdida de calidad organoléptica y nutricional (TomásBarberán y Espín, 2001, Jeong et al., 2008).

La papa presenta una velocidad de pardeamiento oxidativo alta, por lo que estudiar antioxidantes que redujesen o inhibiesen esta reacción, podría ser un referente en el control de las reacciones bioquímicas causantes del pardeamiento para otras hortalizas. Varios estudios mostraron la relación entre el genotipo y la susceptibilidad al pardeamiento enzimático y esto fue explicado por la diferencia en el contenido de fenoles y antioxidantes y por la actividad enzimática (Cabezas-Serrano et al., 2009). 
Los compuestos químicos derivados del azufre son ampliamente empleados entre los distintos agentes antipardeamiento (Laurila et al., 1998). Sin embargo su utilización en productos de papa sólo es permitida a bajas concentraciones dado que pueden causar reacciones alérgicas en personas sensibles a este compuesto (FDA, 1994), además de sabores indeseables y una disminución importante en el valor nutricional del producto (Chalom et al., 1995). Para reemplazar el uso de metabisulfito de sodio (MS) se planteó estudiar agentes antipardeamiento, considerados naturales y saludables para el consumidor, como el ácido ascórbico (AA) y el ácido cítrico (AC). El AA se considera un inhibidor altamente efectivo del pardeamiento enzimático, debido a su capacidad de reducir las quinonas, generadas por la oxidación de los polifenoles, hacia los compuestos fenólicos (incoloros) (Oms-Oliu et al., 2006). No obstante una vez que el ácido ascórbico agregado ha sido completamente oxidado a ácido dehidroascórbico, las quinonas pueden acumularse y se polimerizan, originando el pardeamiento (Friedman, 1997). Debido a esto se utiliza el AA combinado con otro agente, como puede ser el ácido cítrico, y así aumentar la eficacia individual por la diferente forma de acción en la inhibición del proceso de oscurecimiento (Limbo y Piergiovanni, 2006, 2007; Rocculi et al., 2007). En este trabajo se propuso sustituir o reducir el uso de metabisulfito en PMP de dos variedades de papa, Spunta y Newen INTA, almacenados durante 14 días a $4^{\circ} \mathrm{C}$, con la aplicación de diferentes concentraciones y combinaciones de antioxidantes ácido cítrico y ácido ascórbico para mantener la calidad de los PMP durante su almacenamiento a $4^{\circ} \mathrm{C}$

\section{Materiales y métodos}

\section{Material Vegetal}


Se estudiaron dos variedades de papa (Solanum tuberosum L.) con diferente susceptibilidad al pardeamiento enzimático. La variedad Newen INTA, originada en el Programa de Mejoramiento de Papa, de la Estación Experimental Agropecuaria INTA, Balcarce y caracterizada como susceptible al pardeamiento enzimático; y la variedad Spunta de mayor superficie de cultivo en la Argentina y la principal oferta para el consumo fresco. Ambas, fueron cultivadas en campos de la Estación Experimental Agropecuaria INTA, Balcarce, Sudeste de la provincia de Buenos Aires (3750'47', S, 58 $15^{\prime} 19^{\prime}$ ' O) y luego de la cosecha se mantuvieron en cámara frigorífica a $4^{\circ} \mathrm{C}$ hasta el inicio del ensayo.

\section{Muestreo y preparación del material vegetal}

Se tomó una muestra de $5 \mathrm{~kg}$. de tubérculos de tamaño mediano y libres de defectos externos. Los tubérculos fueron lavados con agua potable y sumergidos en una solución de hipoclorito de sodio (100ppm) con agitación por $5 \mathrm{~min}$. Se pelaron y cortaron en forma de bastones; se eligieron 9 bastones centrales por tubérculo y se lavaron con agua potable. Los bastones fueron escurridos y luego inmersos en las soluciones ensayadas como tratamientos, con una relación (peso/volumen) de 1:10 durante $3 \mathrm{~min}$. Trascurrido el tiempo, los bastones se escurrieron, y se envasaron en bolsas de polietileno de baja densidad, conteniendo aproximadamente 300 gr. de muestra cada bolsa. Las mismas se sellaron manualmente y se almacenaron en cámaras a $4^{\circ} \mathrm{C}$.

\section{Tratamientos}

Las concentraciones de los agentes antipardeantes se definieron por ensayos preliminares y teniendo en cuenta trabajos anteriores (Ayerdi et al., 2005; Limbo y Piergiovanni, 2006). 
Para Newen INTA se ensayaron los tratamientos: T 0: testigo; T 1: MS 0,1\%; T 2: AA 1\% + AC 1\%; T 3: AA 0,5\%+AC 0,1\%; T 4: AA 1,5\% + AC 0,5\%.

Para Spunta, se ensayaron los tratamientos: T 0: testigo; T 1: MS 0,1\%; T 2: AA $1 \%+\mathrm{AC}$ 1\%; T 3: AA 1,5\% + AC 0,5\%; T 4: MS 0,05\% + AA 1\%; T 5: MS 0,05\% + AC 0,5\%; T 6: MS $0,05 \%+\mathrm{AA} 1 \%+\mathrm{AC} 0,5 \%$.

Todos los tratamientos contemplaron dosis de $0,02 \%$ de sorbato de potasio para controlar el desarrollo microbiano.

\section{Determinaciones}

Los análisis químicos y organolépticos (luminosidad del color) en los PMP de papa fueron realizados a los $1,4,7,10$ y 14 días de almacenamiento a $4^{\circ} \mathrm{C}$.

\section{Fenoles totales}

El contenido de fenoles totales se determinó por el método espectrofotométrico de FolinCiocalteu descripto por Blessington (2005) con modificaciones. Los resultados se expresaron en $\mu \mathrm{g}$ de ácido gálico equivalente por gramo de peso fresco ( $\mu \mathrm{g}$ AGE/ g pf).

\section{Capacidad antioxidante total}

La capacidad antioxidante total (CAT) se determinó mediante el método del DPPH (1,1difenil-2-picrlhidrazil) descripto por Brand-Williams et al. (1995). Se construyó una curva de calibración con una solución de Trolox $0.5 \mathrm{mM}$ y los resultados fueron expresados en $\mu \mathrm{g}$ Trolox equivalentes por gramo peso fresco ( $\mu \mathrm{g}$ Trolox E/ g pf).

\section{Ácido ascórbico}


La concentración de ácido ascórbico (AA) se determinó por HPLC (High Performance Liquid Chromatography) de acuerdo a la metodología de Tudela et al. (2002). Se construyó una curva estándar de ácido ascórbico (Bio Pack) y los resultados fueron expresados en mg AA en $100 \mathrm{~g}$ de peso fresco (mg AA/100 g pf).

\section{Color. Luminosidad}

Para la determinación de color se empleó un colorímetro triestímulo Minolta modelo CR300 (Whitaker y Lee, 1995). Se tuvo en cuenta el Valor L (luminosidad) para estimar el pardeamiento.

\section{Actividad de la polifenoloxidasa}

Para la determinación de la actividad de la PPO se pesaron 5 g de papa recién triturada, se mezcló con buffer fosfato de sodio $0.1 \mathrm{M}$, se homogeneizó durante 1 minuto a $11.000 \mathrm{rpm}$, filtró y centrifugó durante 15 minutos a $5000 \mathrm{rpm}$. Una alícuota $(1 \mathrm{~mL})$ del sobrenadante se agregó sobre una solución catecol al 0,1\%. Se leyó la absorbancia con intervalos de $5 \mathrm{~s}$ durante un minuto en longitud de onda de $410 \mathrm{~nm}$. Se graficó el cambio en la absorbancia respecto al tiempo. Se expresó la actividad enzimática como unidad de actividad de la enzima PPO (UPPO), definida como la cantidad de enzima que causa un cambio en la absorbancia de $0.1 \mathrm{~nm}$ por min.

\section{Diseño experimental y análisis estadístico}

Se utilizó un Diseño Completamente Aleatorizado con 3 repeticiones para cada variedad y tiempo de almacenamiento. Se realizó el análisis de varianza ANOVA y se compararon las 
medias con el test de Tukey con un $\alpha=0,05$. Además, se efectuó un análisis de correlación (Conover, 1999). Los datos estadísticos fueron analizados con el programa InfoStat (2011).

\section{Resultados y discusión}

El efecto en el contenido de fenoles totales (FT) y ácido ascórbico (AA), la capacidad antioxidante total (CAT), la actividad de la enzima PPO y la luminosidad (L) de los agentes antipardeamiento aplicados a PMP de las variedades Newen INTA y Spunta se muestran en la Tabla 1 a y b.

Tabla 1 a. El efecto de los agentes antipardeamiento, en el contenido de FT y AA, CAT, la actividad de la PPO y el valor $\mathrm{L}$ de los PMP a los 0 y 10 días de almacenamiento a $4^{\circ} \mathrm{C}$ en Newen INTA.

\begin{tabular}{|c|c|c|c|c|c|c|c|c|c|c|}
\hline \multirow[b]{2}{*}{ Tratamiento } & \multicolumn{2}{|c|}{ AA (mg AA/100 g pf) } & \multicolumn{2}{|c|}{ FENOLES ( $\mu \mathrm{g}$ AGE/g pf) } & \multicolumn{2}{|c|}{ CAT ( $\mu$ g TroloxE/g pf) } & \multicolumn{2}{|c|}{$\begin{array}{c}\begin{array}{c}\text { Actividad de PPO } \\
\text { (UPPO) }\end{array} \\
\end{array}$} & \multicolumn{2}{|c|}{ Luminosidad (Valor $\mathbf{L}$ ) } \\
\hline & 0 día & 10 días & 0 día & 10 días & 0 día & 10 días & 0 día & 10 días & 0 día & 10 días \\
\hline Testigo (T0) & $12,7 \pm 0,3^{\mathrm{A}}$ & $10,5 \pm 0,4^{\mathrm{B}}$ & $373,7 \pm 4,8^{\mathrm{A}}$ & $317,6 \pm 8,5^{\mathrm{B}}$ & $89,2 \pm 1,4^{\mathrm{A}}$ & $72,1 \pm 1,6^{\mathrm{B}}$ & $23,5 \pm 0,2^{\mathrm{B}}$ & $27,1 \pm 0,4^{\mathrm{A}}$ & $66,1 \pm 0,2^{\mathrm{A}}$ & $56,5 \pm 0,8^{\mathrm{B}}$ \\
\hline $\begin{array}{c}\text { MS 0,1 \% } \\
\text { (T1) }\end{array}$ & $3,4 \pm 0,1^{\mathrm{B}}$ & $4,1 \pm 0,1^{\mathrm{A}}$ & $356,8 \pm 20,2^{\mathrm{B}}$ & $449,2 \pm 12,2^{\mathrm{A}}$ & $90,3 \pm 2,8^{\text {B }}$ & $88,5 \pm 1,7^{\mathrm{B}}$ & $20,0 \pm 1,4^{\mathrm{A}}$ & $20,6 \pm 0,9^{\mathrm{A}}$ & $70,5 \pm 0,9^{\mathrm{A}}$ & $72,0 \pm 0,5^{\mathrm{A}}$ \\
\hline $\begin{array}{c}\text { AA 1\% + AC } \\
1 \% \text { (T2) }\end{array}$ & $50,0 \pm 0,5^{\mathrm{A}}$ & $28,1 \pm 0,1^{\mathrm{B}}$ & $712,7 \pm 162,0^{\mathrm{AB}}$ & $1063,4 \pm 86,0^{\mathrm{A}}$ & $174,7 \pm 4,3^{\mathrm{A}}$ & $118,9 \pm 0,9^{\mathrm{B}}$ & $22,9 \pm 2,2^{\mathrm{A}}$ & $25,0 \pm 1,7^{\mathrm{A}}$ & $67,4 \pm 0,6^{\mathrm{A}}$ & $63,1 \pm 0,1^{\mathrm{B}}$ \\
\hline $\begin{array}{c}\text { AA 0,5\% + } \\
\text { AC 0,1\% } \\
\text { (T3) }\end{array}$ & $18,2 \pm 0,1^{\mathrm{B}}$ & $26,1 \pm 0,0^{\mathrm{A}}$ & $640,2 \pm 57,6^{\mathrm{AB}}$ & $804,6 \pm 69,2^{\mathrm{A}}$ & $82,0 \pm 4,2^{\mathrm{B}}$ & $117,4 \pm 2,5^{\mathrm{A}}$ & $26,6 \pm 0,2^{\mathrm{A}}$ & $23,1 \pm 0,7^{\mathrm{B}}$ & $66,1 \pm 0,5^{\mathrm{A}}$ & $60,8 \pm 0,9^{\mathrm{B}}$ \\
\hline $\begin{array}{c}\text { AA 1,5\% + } \\
\text { AC 0,5\% } \\
\text { (T4) }\end{array}$ & $47,6 \pm 0,3^{\mathrm{A}}$ & $35,4 \pm 0,2^{\mathrm{B}}$ & $976,7 \pm 15,4^{\mathrm{A}}$ & $950,6 \pm 24,4^{\mathrm{A}}$ & $412,2 \pm 1,1^{\mathrm{A}}$ & $162,3 \pm 11,1^{\mathrm{B}}$ & $21,1 \pm 0,1^{\mathrm{A}}$ & $23,1 \pm 0,2^{\mathrm{A}}$ & $69,1 \pm 1,0^{\mathrm{A}}$ & $65,3 \pm 0,5^{\mathrm{A}}$ \\
\hline
\end{tabular}

Letras diferentes en la misma fila, dentro de cada determinación, indican diferencias significativas por el test de Tukey $(\alpha \geq 0.05)$.

*Cada valor es expresado como la media \pm desviación estándar. 
Tabla 1 b. El efecto de los agentes antipardeamiento, en el contenido de FT y AA, CAT, la actividad de la PPO y el valor L de los PMP a los 0 y 10 días de almacenamiento a $4^{\circ} \mathrm{C}$ en Spunta.

\begin{tabular}{|c|c|c|c|c|c|c|c|c|c|c|}
\hline \multirow[b]{2}{*}{ Tratamiento } & \multicolumn{2}{|c|}{ AA (mg AA/100 g pf) } & \multicolumn{2}{|c|}{ FENOLES ( $\mu \mathrm{g} \mathrm{AGE/g} \mathrm{pf)}$} & \multicolumn{2}{|c|}{ CAT ( $\mu$ g TroloxE/g pf) } & \multicolumn{2}{|c|}{$\begin{array}{c}\begin{array}{c}\text { Actividad de PPO } \\
\text { (UPPO) }\end{array} \\
\end{array}$} & \multicolumn{2}{|c|}{ Luminosidad (Valor L) } \\
\hline & 0 día & 10 días & 0 día & 10 días & 0 día & 10 días & 0 día & 10 días & 0 día & 10 días \\
\hline Testigo (T0) & $3,1 \pm 0,9^{\mathrm{A}}$ & $3,2 \pm 0,3^{\mathrm{A}}$ & $69,8 \pm 1,3^{\mathrm{A}}$ & $59,2 \pm 2,9^{\mathrm{A}}$ & $71,4 \pm 8,0^{\mathrm{A}}$ & $79,8 \pm 2,3^{\mathrm{A}}$ & $4,4 \pm 0,4^{\mathrm{A}}$ & $5,0 \pm 0,9^{\mathrm{A}}$ & $68,7 \pm 0,2^{\mathrm{A}}$ & $64,2 \pm 2,0^{\mathrm{B}}$ \\
\hline $\begin{array}{c}\text { MS 0,1 \% } \\
\text { (T1) }\end{array}$ & $6,7 \pm 0,6^{\mathrm{A}}$ & $4,8 \pm 0,5^{\mathrm{AB}}$ & $62,5 \pm 0,6^{\mathrm{A}}$ & $66,4 \pm 0,7^{\mathrm{A}}$ & $108,0 \pm 3,9^{\mathrm{A}}$ & $98,6 \pm 8,2^{\mathrm{B}}$ & $4,4 \pm 1,0^{\mathrm{A}}$ & $5,4 \pm 1,0^{\mathrm{A}}$ & $70,1 \pm 0,6^{\mathrm{A}}$ & $69,1 \pm 1,6^{\mathrm{A}}$ \\
\hline $\begin{array}{c}\text { AA 1\% + AC } \\
1 \% \text { (T2) }\end{array}$ & $67,7 \pm 1,5^{\mathrm{A}}$ & $18,8 \pm 2,1^{\mathrm{B}}$ & $129,8 \pm 6,6^{\mathrm{A}}$ & $109,1 \pm 0,8^{\mathrm{B}}$ & $530,9 \pm 11,5^{\mathrm{A}}$ & $130,0 \pm 10,0^{\mathrm{B}}$ & $4,6 \pm 0,4^{\mathrm{A}}$ & $5,6 \pm 0,4^{\mathrm{A}}$ & $69,5 \pm 0,2^{\mathrm{A}}$ & $65,7 \pm 0,1^{\mathrm{B}}$ \\
\hline $\begin{array}{c}\text { AA 1,5\% + } \\
\text { AC 0,5\% } \\
\text { (T3) }\end{array}$ & $92,2 \pm 2,3^{\mathrm{A}}$ & $23,1 \pm 2,4^{\mathrm{B}}$ & $153,2 \pm 5,4^{\mathrm{A}}$ & $111,7 \pm 6,9^{\mathrm{B}}$ & $556,1 \pm 2,2^{\mathrm{A}}$ & $155,7 \pm 11,8^{\text {B }}$ & $1,6 \pm 1,6^{\mathrm{A}}$ & $3,4 \pm 0,2^{\mathrm{A}}$ & $70,3 \pm 0,4^{\mathrm{A}}$ & $68,2 \pm 0,2^{\mathrm{A}}$ \\
\hline $\begin{array}{l}\text { MS } 0,05 \%+ \\
\text { AA 1\% (T4) } \\
\end{array}$ & $57,6 \pm 3,4^{\mathrm{A}}$ & $16,6 \pm 0,1^{\mathrm{B}}$ & $139,0 \pm 3,4^{\mathrm{A}}$ & $119,3 \pm 3,9^{\mathrm{B}}$ & $623,8 \pm 20,9^{\mathrm{A}}$ & $360,8 \pm 3,2^{\mathrm{B}}$ & $0,2 \pm 0,2^{\mathrm{A}}$ & $0,0 \pm 0,0^{\mathrm{A}}$ & $71,0 \pm 0,3^{\mathrm{A}}$ & $67,8 \pm 0,2^{\mathrm{B}}$ \\
\hline $\begin{array}{c}\text { MS 0,05\% + } \\
\text { AC 0,5\% } \\
\text { (T5) } \\
\end{array}$ & $6,1 \pm 0,1^{\mathrm{A}}$ & $5,6 \pm 0,0^{\mathrm{A}}$ & $75,0 \pm 1,8^{\mathrm{A}}$ & $74,9 \pm 1,3^{\mathrm{A}}$ & $193,5 \pm 6,1^{\mathrm{A}}$ & $213,4 \pm 3,4^{\mathrm{A}}$ & $0,0 \pm 0,0^{\mathrm{A}}$ & $0,2 \pm 0,2^{\mathrm{A}}$ & $69,6 \pm 0,3^{\mathrm{A}}$ & $67,2 \pm 0,0^{\mathrm{B}}$ \\
\hline $\begin{array}{c}\text { MS 0,05\% + } \\
\text { AA 1\% + AC } \\
0,5 \% \text { (T6) }\end{array}$ & $59,8 \pm 0,6^{\mathrm{A}}$ & $16,3 \pm 0,6^{\mathrm{B}}$ & $134,1 \pm 0,4^{\mathrm{A}}$ & $123,9 \pm 4,0^{\mathrm{B}}$ & $630,6 \pm 11,6^{\mathrm{A}}$ & $393,7 \pm 8,3^{\mathrm{B}}$ & $0,0 \pm 0,0^{\mathrm{A}}$ & $0,0 \pm 0,0^{\mathrm{A}}$ & $71,4 \pm 0,6^{\mathrm{A}}$ & $68,5 \pm 0,3^{\mathrm{B}}$ \\
\hline
\end{tabular}

Letras diferentes en la misma fila, dentro de cada determinación, indican diferencias significativas por el test de Tukey $(\alpha \geq 0.05)$.

*Cada valor es expresado como la media \pm desviación estándar.

\section{Fenoles Totales}
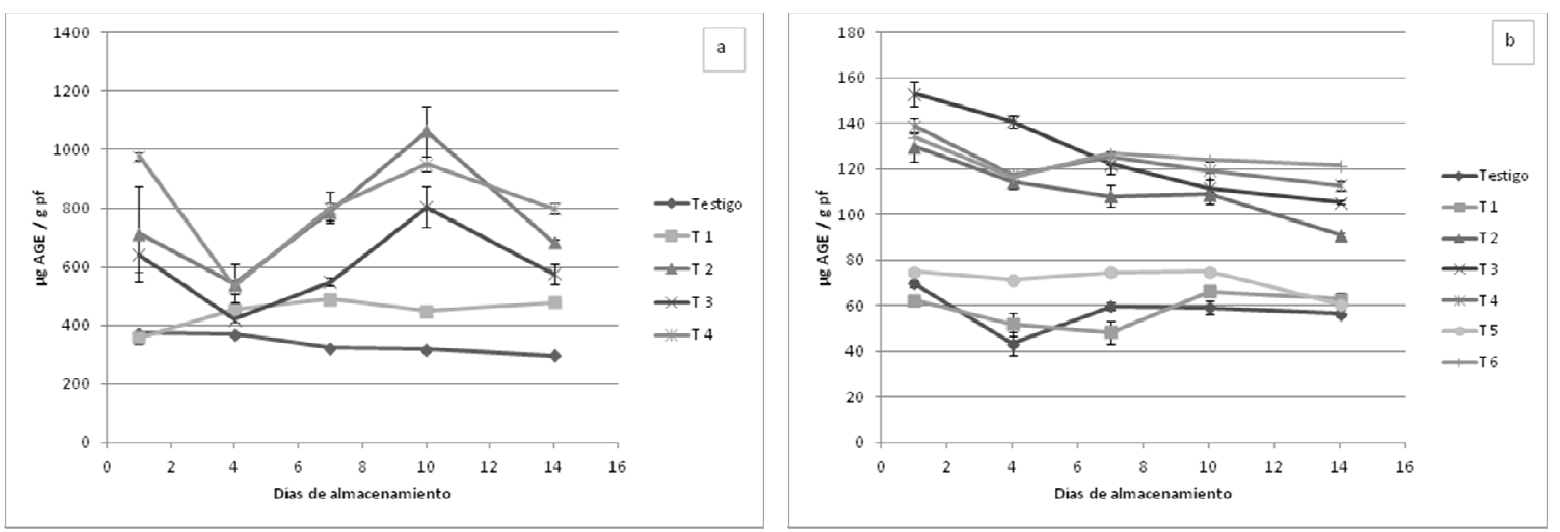

Figura 1 a y b. Variación en el contenido de FT ( $\mu$ g AGE/g pf) durante el almacenamiento a $4{ }^{\circ} \mathrm{C}$ en cortes de papa de las variedades Newen INTA (a) y Spunta (b) tratados con agentes antioxidantes. a- $($ T $0=$ testigo; $\mathrm{T} 1=$ MS 0,1 \%; T $2=\mathrm{AA} 1 \%+\mathrm{AC} 1 \%$; T $3=\mathrm{AA} 0,5 \%+\mathrm{AC} 0,1 \%$; T $4=\mathrm{AA} 1,5 \%+\mathrm{AC} 0,5 \%)$. b- $(\mathrm{T} 0=$ testigo; $\mathrm{T} 1=\mathrm{MS} 0,1 \% ; \mathrm{T} 2=\mathrm{AA} 1 \%+\mathrm{AC} 1 \% ; \mathrm{T} 3=\mathrm{AA} 1,5 \%+\mathrm{AC} 0,5 \% ; \mathrm{T} 4=\mathrm{MS} 0,05 \%+\mathrm{AA} 1 \% ; \mathrm{T} 5=\mathrm{MS}$ $0,05 \%+\mathrm{AC} 0,5 \% ; \mathrm{T} 6=\mathrm{MS} 0,05 \%+\mathrm{AA} 1 \%+\mathrm{AC} 0,5 \%)$

Los FT mostraron variaciones durante el almacenamiento debido a los tratamientos. En el tratamiento con MS (T 1) presentó en el inicio una concentración de fenoles similar al testigo 
(T 0) y los valores se mantuvieron bajos durante el almacenamiento para ambas variedades. En los tratamientos que combinan AA y AC se observaron valores mayores de FT en el inicio del almacenamiento, para ambas variedades (Figura 1 a y b). Los PMP de la variedad Spunta mostraron valores altos de FT con la combinación de AA y MS (T 4) y no presentaron modificaciones notables en el periodo de almacenamiento. Según Gil et al. (1998), la acción reductora del AA agregado, podría disminuir la degradación de los FT durante el almacenamiento en respuesta a la aplicación de AA en rodajas de manzanas. Por otro lado, el agregado de AA influiría en los valores resultantes de la determinación de los fenoles (Waterhouse, 2002) incrementando su contenido, esto se debe al método de cuantificación espectrofotométrico utilizado, dado que el reactivo de Folin-Ciocalteau reacciona con todos los oxidantes presentes.

Además, este comportamiento de los FT coinciden con los resultados obtenidos por Zhu et al. (2009) en el control del pardeamiento enzimático de pera con AA.

\section{Capacidad antioxidante total}
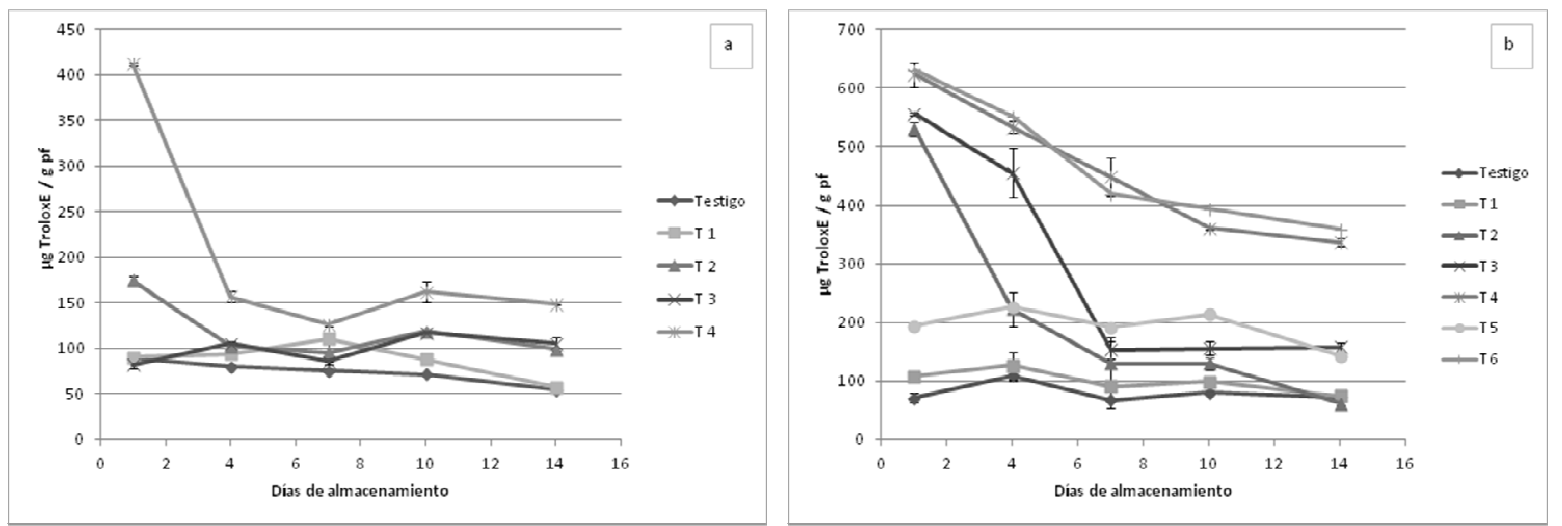

Figura 2 a y b. Variación en la Capacidad antioxidante total $\left(\mu \mathrm{g}\right.$ TroloxE/g pf) durante el almacenamiento a $4^{\circ} \mathrm{C}$ en cortes de papa de las variedades Newen INTA (a) y Spunta (b), tratados con agentes antioxidantes a- $($ T $0=$ testigo; T $1=$ MS 0,1 \%; T $2=$ AA $1 \%+$ AC $1 \%$; T $3=$ AA $0,5 \%+$ AC 0,1\%; T $4=$ AA $1,5 \%+$ AC $0,5 \%)$. b$(\mathrm{T} 0=$ testigo; $\mathrm{T} 1=\mathrm{MS} 0,1 \% ; \mathrm{T} 2=\mathrm{AA} 1 \%+\mathrm{AC} 1 \% ; \mathrm{T} 3=\mathrm{AA} 1,5 \%+\mathrm{AC} 0,5 \% ; \mathrm{T} 4=\mathrm{MS} 0,05 \%+\mathrm{AA}$ $1 \% ;$ T $5=\operatorname{MS~} 0,05 \%+\mathrm{AC} 0,5 \% ;$ T $6=$ MS $0,05 \%+\mathrm{AA} 1 \%+\mathrm{AC} 0,5 \%)$ 
La CAT de los tratamientos con agregado de AA fue significativamente mayor con respecto a los PMP que no lo contenían. A su vez, el tratamiento solo con MS (T 1), mantuvo muy baja CAT en todo el almacenamiento, no diferenciándose del testigo, en ambas variedades (Figura 2 a y b). Por otro lado, en los PMP de la variedad Spunta se observó que la adición de AA junto con una baja concentración de MS, con o sin AC (T 4 y T 6), mantuvo la mayor CAT durante todo el período. Estos resultados sugieren que el MS no afecta la CAT, y el efecto antioxidante se atribuiría solamente al agregado de AA. Estos resultados son similares a los de Cocci et al. (2006) quien obtuvo valores de capacidad antioxidante más altos en las muestras inmersas en AA comparadas con un testigo, probablemente por la mayor concentración de AA. La CAT mostró una correlación positiva con el contenido de AA y con los FT. La correlación entre CAT y contenido de AA fue de 0.87 en el T 2 y de 0.80 en el T 4. En el T 4 se encontró la mayor correlación entre la CAT y los FT (r=0.51). 


\section{Ácido ascórbico}
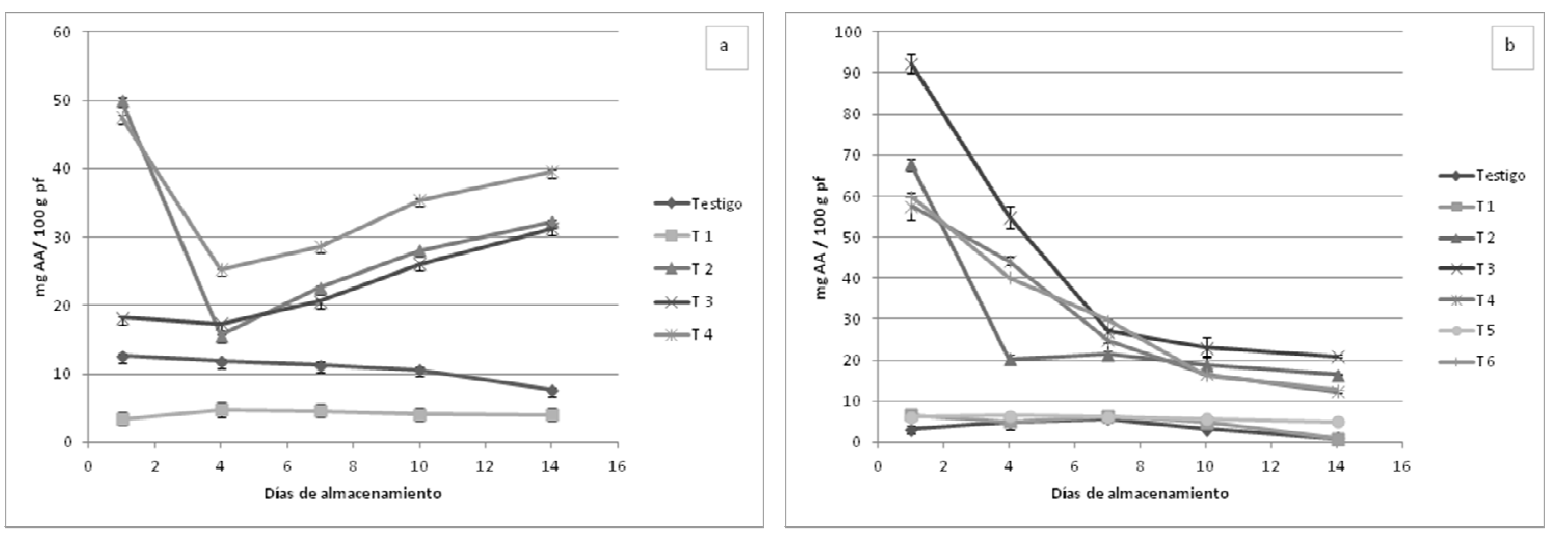

Figura 3 a y b. Variación en el contenido de ácido ascórbico (mg AA/100 g pf) durante el almacenamiento a $4^{\circ} \mathrm{C}$ en cortes de papa de las variedades Newen INTA (a) y Spunta (b) tratados con agentes antioxidantes. a- (T $0=$ testigo; $1=\operatorname{MS~} 0,1 \% ; \mathrm{T} 2=\mathrm{AA} 1 \%+\mathrm{AC} 1 \% ; \mathrm{T} 3=\mathrm{AA} 0,5 \%+\mathrm{AC} 0,1 \% ; \mathrm{T} 4=\mathrm{AA} 1,5 \%+\mathrm{AC} 0,5 \%)$. b- $(\mathrm{T} 0=$ testigo; $\mathrm{T} 1=\mathrm{MS} 0,1 \% ; \mathrm{T} 2=\mathrm{AA} 1 \%+\mathrm{AC} 1 \% ; \mathrm{T} 3=\mathrm{AA} 1,5 \%+\mathrm{AC} 0,5 \% ; \mathrm{T} 4=\mathrm{MS} 0,05 \%+\mathrm{AA}$ $1 \% ; \mathrm{T} 5=\mathrm{MS} 0,05 \%+\mathrm{AC} 0,5 \% ; \mathrm{T} 6=\mathrm{MS} 0,05 \%+\mathrm{AA} 1 \%+\mathrm{AC} 0,5 \%)$.

En Newen INTA, el contenido de AA en las papas tratadas con MS (T 1) fue menor al de la papa sin tratar durante todo el almacenamiento. Lo que podría significar una pérdida en el valor nutricional y coincide con lo observado por Chalom et al. (1995). Los tratamientos con concentraciones mayores de AA y AC (T 2 y T 4), mostraron inicialmente, valores de AA de hasta 4 veces más altos que las muestras sin tratar (Figura 3 a). El tratamiento con dosis bajas de AA y AC (T 3) también presentó mayor contenido de AA que el testigo, pero menor que T 2 y T 4. Estos valores decrecieron en el cuarto día de almacenamiento en un 50-70\% para el T 4 y $\mathrm{T} 2$ respectivamente. En los tratamientos con AA y AC (T 2, T 3 y T 4) se encontró un aumento no explicable en la concentración de AA durante el período de almacenamiento y estos valores fueron mayores que en el tratamiento con MS (T 1).

En Spunta, los tratamientos con MS y MS con AA (T 1 y T 5) mantuvieron el contenido de AA con respecto al testigo hasta el décimo día de almacenamiento. En las papas tratadas solo con AA y AC (T 2 y T 3), se observaron inicialmente valores de AA mayores que el resto de 
los tratamientos, para luego descender significativamente en el cuarto día de almacenamiento a un 60 y $70 \%$ para el T 3 y T 2 respectivamente (Figura 3 b).

Si bien se observó un descenso significativo de AA durante el almacenamiento, su contenido resultó siempre mayor en los tratamientos con altas concentraciones de AA y AC con respecto al testigo. Estos resultados son semejantes a los de Cocci et al. (2006) que encontró contenidos de AA significativamente más altos en manzanas tratadas con AA comparadas con muestras sin tratar. La disminución de la concentración de AA se debería a su función como antioxidante, debido a la cual mantiene la concentración de FT con valores similares entre el inicio y el final del almacenamiento. El agregado de AA en este tipo de tratamiento podría ser un camino para enriquecer el tejido vegetal contribuyendo a un incremento en la capacidad antioxidante que normalmente deriva de los compuestos endógenos bioactivos.

\section{Color}

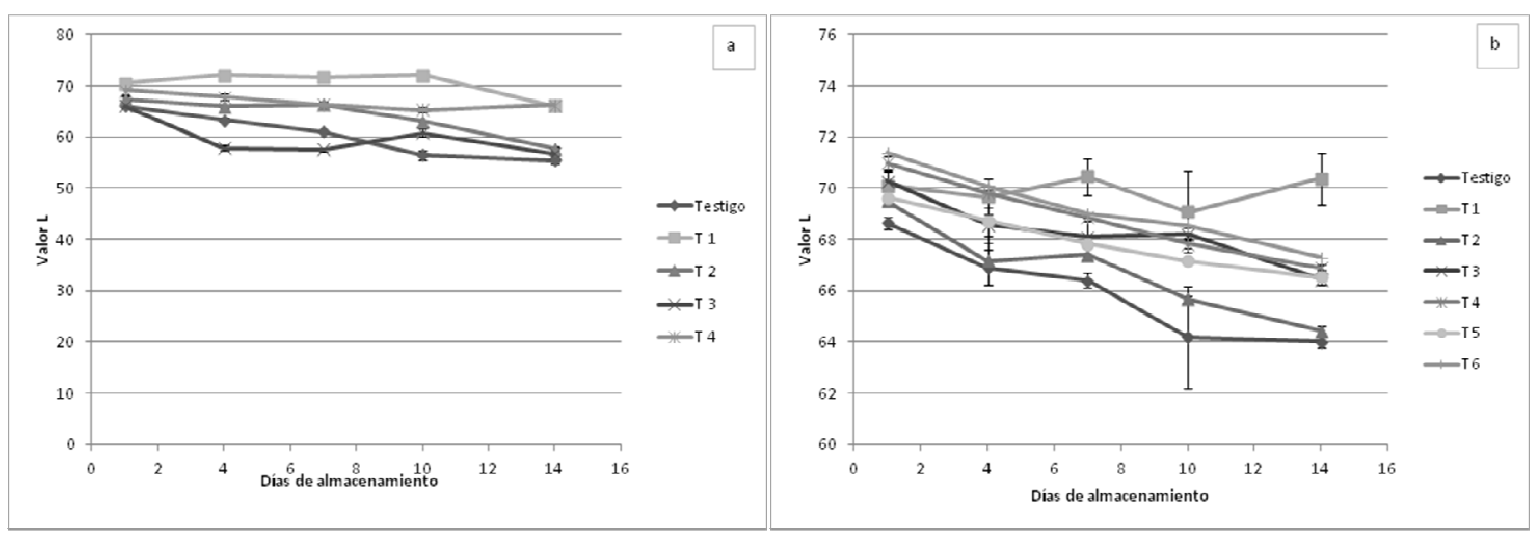

Figura 4 a y b. Variación en el Luminosidad (L) durante el almacenamiento a $4^{\circ} \mathrm{C}$ en cortes de papa de las variedades Newen INTA (a) y Spunta (b) tratados con agentes antioxidantes. a- (T $0=$ testigo; T $1=$ MS 0,1 \%; $\mathrm{T} 2=\mathrm{AA} 1 \%+\mathrm{AC} 1 \% ; \mathrm{T} 3=\mathrm{AA} 0,5 \%+\mathrm{AC} 0,1 \% ; \mathrm{T} 4=\mathrm{AA} 1,5 \%+\mathrm{AC} 0,5 \%) \cdot \mathrm{b}-(\mathrm{T} 0=$ testigo; $\mathrm{T} 1=\mathrm{MS}$ $0,1 \% ; \mathrm{T} 2=\mathrm{AA} 1 \%+\mathrm{AC} 1 \% ; \mathrm{T} 3=\mathrm{AA} 1,5 \%+\mathrm{AC} 0,5 \% ; \mathrm{T} 4=\mathrm{MS} 0,05 \%+\mathrm{AA} 1 \% ; \mathrm{T} 5=\mathrm{MS} 0,05 \%+\mathrm{AC}$ $0,5 \% ;$ T $6=$ MS $0,05 \%+\mathrm{AA} 1 \%+\mathrm{AC} 0,5 \%)$. 
En Newen INTA, el agregado de MS (T 1) resultó en un mayor valor de luminosidad, el cual se mantuvo constante, disminuyendo al final del almacenamiento. El valor L en los tratamientos con AA y AC, fue mayor en el tratamiento con las mayores dosis de AA y AC (T 4) y no mostró diferencias durante el almacenamiento. El T 2 presentó una disminución significativa en el día 10 y el tratamiento con menores dosis (T 3), a partir del cuarto día (Figura 4 a).

En la variedad Spunta, el testigo mostró mayor oscurecimiento con respecto al resto de los tratamientos. Los PMP tratados con MS mantuvieron el color inicial durante todo el periodo de almacenamiento y no fueron superados por ningún otro tratamiento. El tratamiento con dosis bajas de AA y AC (T 2) disminuyó el valor L significativamente durante el almacenamiento, mientras que los tratamientos con MS combinado tanto con AA como con $\mathrm{AC}$ ( $\mathrm{T} 4$ y $\mathrm{T}$ 5) presentaron al inicio valores altos de luminosidad que disminuyeron significativamente durante el periodo de almacenamiento. Los tratamientos $\mathrm{T} 4$ y $\mathrm{T}$ 6, a pesar que su valores de L disminuyeron a lo largo de todo el almacenamiento, no presentaron diferencias con respecto a los PMP tratados con MS (T 1) hasta el décimo día (Figura 4 b).

\section{Actividad de la polifenoloxidasa}

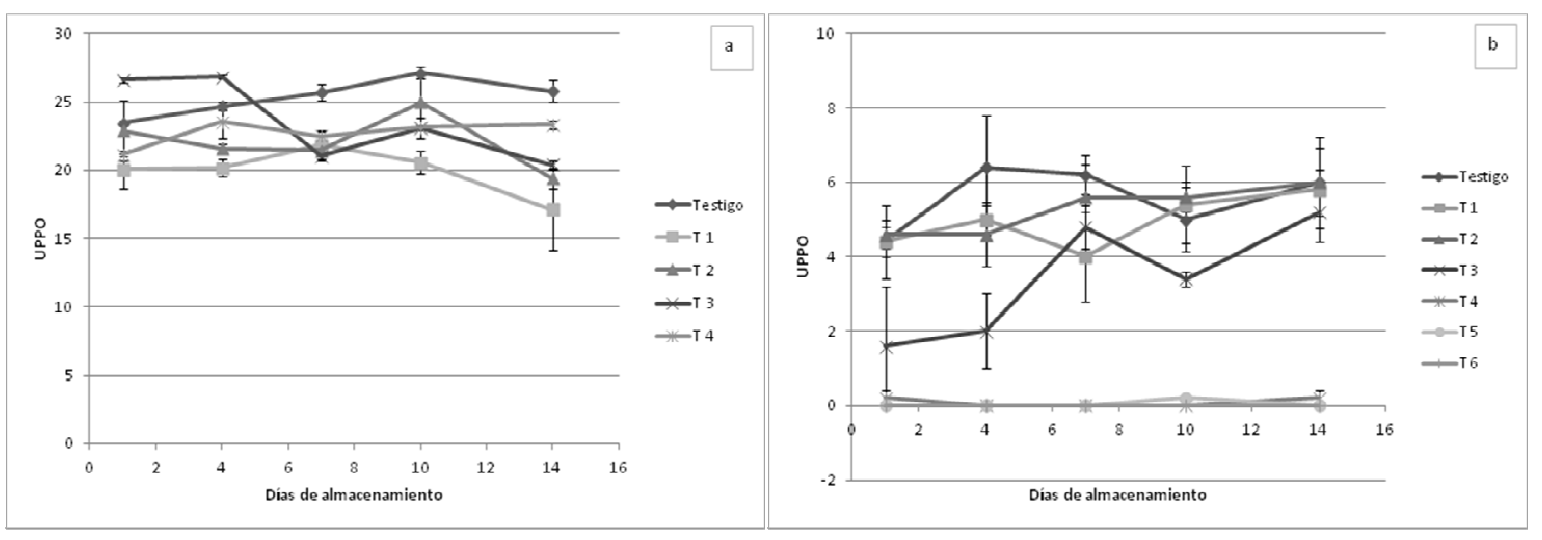

Figura 5 a y b. Variación en la actividad de la PPO (UPPO) durante el almacenamiento a $4{ }^{\circ} \mathrm{C}$ en cortes de papa de las variedades Newen INTA (a) y Spunta (b) tratados con agentes antioxidantes. a- $(\mathrm{T} 0=$ testigo; $\mathrm{T} 1=\mathrm{MS}$ 
$0,1 \% ; \mathrm{T} 2=\mathrm{AA} 1 \%+\mathrm{AC} 1 \% ; \mathrm{T} 3=\mathrm{AA} 0,5 \%+\mathrm{AC} 0,1 \% ; \mathrm{T} 4=\mathrm{AA} 1,5 \%+\mathrm{AC} 0,5 \%) \cdot \mathrm{b}-(\mathrm{T} 0=$ testigo; $\mathrm{T} 1$ $=\operatorname{MS~} 0,1 \% ; \mathrm{T} 2=\mathrm{AA} 1 \%+\mathrm{AC} 1 \% ; \mathrm{T} 3=\mathrm{AA} 1,5 \%+\mathrm{AC} 0,5 \% ; \mathrm{T} 4=\mathrm{MS} 0,05 \%+\mathrm{AA} 1 \% ; \mathrm{T} 5=\mathrm{MS}$ $0,05 \%+\mathrm{AC} 0,5 \% ; \mathrm{T} 6=\mathrm{MS} 0,05 \%+\mathrm{AA} 1 \%+\mathrm{AC} 0,5 \%)$

Se observó una alta actividad de la PPO desde los primeros días de almacenamiento, en la variedad Newen INTA. Las combinaciones de altas concentraciones de AA y AC, mantuvieron la actividad de la PPO constante durante el almacenamiento, comportamiento que también se presentó en el tratamiento con MS (Figura 5 a).

Los tratamientos con dosis mínimas de MS en combinación con AA y AC (T 4, T 5 y T 6) en los PMP de la variedad Spunta, mostraron una inhibición total de la actividad de la PPO. En aquellas combinaciones de AA y AC sin MS (T 2 y $\mathrm{T} 3$ ), se registraron valores bajos de actividad de la enzima, principalmente en los primeros días del almacenamiento (Figura $5 \mathrm{~b}$ ). Las bajas concentraciones de AA y AC aumentaron la actividad de la enzima y disminuyeron la luminosidad, resultado que concuerda con el publicado por Sapers et al. (1989), quienes encontraron que la actividad de la PPO estaba correlacionada con el pardeamiento en papas. Sin embargo, lo obtenido por Cantos et al. (2002) no justificó esa correlación.

En general, el tratamiento con MS (T 1), sólo ofrece mayores ventajas en cuanto a mantener el color en los PMP de papa, ya que para ambas variedades, el valor de luminosidad no fue superado por ninguno de los tratamientos. A su vez, no aumenta la cantidad de FT y CAT comparado con los demás tratamientos, si bien mostró un alto poder inhibitorio de la enzima PPO. Es importante destacar que disminuye significativamente el contenido de AA respecto al testigo en los PMP de la variedad Newen INTA y no lo modifica en los de Spunta.

Por otro lado, los tratamientos que combinaron MS con AA y que fueron aplicados a los PMP de la variedad Spunta, mostraron mejores resultados hasta el día 10, en cuanto a una mayor concentración de FT, una mayor CAT, una mayor luminosidad de los PMP, similar al 
tratamiento con MS, y una inhibición total de la enzima PPO en comparación con los demás tratamientos. Sin embargo, si se los relaciona con el tratamiento que contiene $1,5 \%$ de AA y 0,5\% de $\mathrm{AC}$ ( $\mathrm{T}$ 3), se observa que si bien, se mantienen los mismos parámetros de luminosidad, ofrece la ventaja de conservar un mayor contenido de AA hasta el final del almacenamiento.

Un comportamiento similar se observó en la variedad Newen INTA, donde el tratamiento con 1,5\% de AA y $0,5 \%$ de AC (T 4) mostró los mejores resultados en la cantidad de FT, en la CAT hasta el día 10 y en la concentración de AA, si bien no superó al tratamiento con MS en la luminosidad y en la inhibición de la PPO.

De acuerdo a estos resultados, la combinación de 1,5\% de AA y 0,5\% de AC, como antioxidantes, podría reemplazar efectivamente al tratamiento de MS, manteniendo la luminosidad y aumentando la concentración de AA y FT de los PMP de papa de las variedades Newen INTA y Spunta hasta los 10 días de almacenamiento a $4^{\circ} \mathrm{C}$.

La concentración de 0,1\% MS disminuyó la calidad nutricional, en los PMP de la variedad Newen INTA.

En la variedad Spunta, se pudo reducir la concentración de MS a 0,05\% en la combinación con AA, manteniendo la luminosidad y propiedades nutricionales de los PMP de papa hasta los 10 días de almacenamiento a $4^{\circ} \mathrm{C}$.

\section{Conflictos de interés}

Los autores declaran que no existen conflictos de interés.

\section{Literatura citada}

Ayerdi, C.; Arroqui, C.; Virseda, P. 2005. Efecto de la temperatura sobre la tasa de respiración en patata mínimamente procesada tratada con diferentes agentes conservantes. Alimentación, Equipos y Tecnología. 191: 93-96. 
Blessington, T. 2005. The effects of cooking, storage, and ionizing irradiation on Carotenoids, Antioxidant Activity, and Phenolics in Potato (Solanum tuberosum L.). Tesis Master of Science. Texas A\&M University. 39-42.

Brand-Williams, W.; Cuvelier, M.E.; Berset, C., 1995. Use of a free radical method to evaluate antioxidant activity. Lebensmittel- Wissenschaft und Technologie. 28: 25-30.

Cantos, E.; Tudela, J.A.; Gil, M.I.; Espin, J.C. 2002. Phenolic compounds and related enzymes are not rate-limiting in browning development of fresh-cut potatoes. Journal of Agricultural and Food Chemistry. 50: 3015-3023.

Cabezas-Serrano, A.B., Amodio, M.L., Cornacchiab, R., Rinaldi, R.\& Colelli, G. (2009). Suitability of five different potato cultivars (Solanum tuberosum L.) to be processed as freshcut products. Postharvest Biology and Technology, 53, 138-144.

Chalom, S.; Elrezzi, E.; Pena, P.; Astiarsaran, I.; Bello, J. 1995. Composition of sulfited potatoes: comparison with fresh and frozen potatoes. Plant Foods for Human Nutrition. 47: 133-138.

Cocci, E.; Rocculi, P.; Romani, S.; Dalla Rosa, M. 2006. Changes in nutritional properties of minimally processed apples during storage. Postharvest Biology and Technology. 39: 265271.

Conover, W.J. 1999. Practical Nonparametric Statistics. John Wiley \& Sons, Inc., New York.

FDA. 1994. Sulfiting agents: withdrawal of regulation revoking GRAS status for use on "fresh" potatoes served as sold unpackaged and unlabeled to consumers. U.S.Food and Drug Administration. 56:65938-9.

Friedman, M. 1997. Chemistry, biochemistry, and dietary role of potato polyphenols. A review. Journal of Agricultural and Food Chemistry. 45: 1523-1540.

Gil, M.I.; Gorny, J.R.; Kader, A.A. 1998. Postharvest physiology and quality of 'Fuji' apple slices in response to ascorbic acid treatments and low-oxygen atmospheres. HortScience. 33(2): 305-309. 
Jeong, H. L.; Jin, W. J.; Kwang, D. M.; Kee, J. P. 2008. Effects of Anti-Browning Agents on Polyphenoloxidase activity and total phenolics as related to browning of fresh-cut 'Fuji' apple. ASEAN Food Journal. 15(1): 79-87.

Laurila, E.K.; Hurme, E. U.; Ahvenainen, R.T. 1998b. Shelf life of sliced raw potatoes of various cultivar varieties-substitutions of bisulfites. Journal of Food Protection. 64: 13631371.

Limbo, S.; Piergiovanni, L. 2006. Shelf life of minimally processed potatoes. Part 1. Effects of high oxygen partial pressures in combination with ascorbic and citric acids on enzymatic browning. Postharvest Biology and Technology. 39: 254-264.

Limbo, S.; Piergiovanni, L. 2007. Shelf life of minimally processed potatoes. Part 2. Effects of high oxygen partial pressures in combination with ascorbic and citric acids on loss of some quality traits. Postharvest Biology and Technology. 43: 221-229.

Moretti, C. L.; Araújo, A. L.; Marquelli, W. A.; Silva, W. L. C. 2002. Respiratory activity and browning of minimally processed sweet potatoes. Horticultura Brasileira. 20(3): 497-500.

Oms-Oliu, G.; Aguilo-Aguayo, I.; Martin-Belloso, O. 2006. Inhibition of browning on freshcut pear wedges by natural compounds. Journal of Food Science. 71:216-224.

Petri, E.; Arroqui, C.; Angos I.; Virseda, P. 2008. Effect of preservative agents on the respiration rate of minimally processed potato (Solanum tuberosum cv. Monalisa). Journal of Food Science 73 (3):122-126.

Rocculi, P.; Gómez Galindo, F.; Mendoza, F.; Wadso, L.; Romani, S.; Dalla Rosa, M.; SJÖHOLM, I. 2007. Effect of the application of anti-browning substances on the metabolic activity and sugar composition of fresh-cut potatoes. Postharvest Biology and Technology. 43: 151-157.

Tomás-Barberán, F. A.; Espín, J. C. 2001. Phenolic compounds and related enzymes as determinants of quality in fruits and vegetables. Journal Science Food Agriculture. 81:953876.

Tudela, J. A.; Espín, J. C.; Gil, M. I. 2002. Vitamin C retention in fresh-cut potatoes. Postharvest Biology and Technology. 26: 75-84. 
Yurong M.; Qingguo, W.; Gyunghoon, H.; Cantwell, M. 2010. Reassessment of treatments to retard browning of fresh-cut Russet potato with emphasis on controlled atmospheres and low concentrations of bisulphate. International Journal of Food Science and Technology. 45:14861494.

Waterhouse, A.L. 2002. Current Protocols in Food Analytical Chemistry. I1.1.1-I1.1.8.

Whitaker, J.R.; Lee, C.Y. 1995. Recent advances in chemistry of enzymatic browning. ACS Sump. Sr. 600, 2-7.

Zhu, L.Q.; Zhou, J.; Zhu, S.H.; Guo, L.H. 2009. Inhibition of browning on the surface of peach slices by short-term exposure to nitric oxide and ascorbic acid. Food Chemistry. 114: 174-179. 